gene-therapy approach shows promise in the treatment of metastatic human RCC, and suggest that such therapy might complement existing treatments.

Such animal experiments serve as examples of basic science research that show the potential to translate to the bedside. Clinicians should be optimistic that exciting developments in the treatment of RCC are on the horizon.

Original articles Doehn C et al. (2008) Mode-of-action, efficacy, and safety of a homologous multi-epitope vaccine in a murine model for adjuvant treatment of renal cell carcinoma. Eur Urol [doi:10.1016/j.eururo.2008.05.034] Mellon MJ et al. (2008) Suppression of renal cell carcinoma growth and metastasis with sustained antiangiogenic gene therapy. Hum Gene Ther 19: 487-495

\section{GSTM1 implicated in mechanism of broccoli-mediated prevention of prostate cancer}

Traka and colleagues have examined the effects of broccoli consumption on gene expression in the prostate in 21 men with high-grade prostatic intraepithelial neoplasia. The researchers suggest a possible mechanism of action for isothiocyanates, the putative bioactive components of broccoli, in the prevention of prostate carcinogenesis.

Participants were randomly assigned to consume either $400 \mathrm{~g}$ broccoli per week, or $400 \mathrm{~g}$ peas per week, alongside their normal diet for 1 year. Participants were genotyped for GSTM1 (glutathione S-transferase mu 1) because GSTM1-null individuals show a substantially reduced benefit of broccoli consumption; this enzyme may influence release of the isothiocyanate sulforaphane into the plasma. Transrectal ultrasound-guided needle biopsies of the prostate were performed at baseline, at 6 months and at 12 months. At 6 months, differences in prostate gene expression were seen between GSTM1-positive and GSTM1null men only in the broccoli-intervention group. Compared with baseline, more changes in prostate gene expression were seen at 12 months in the broccoli-intervention group than in the pea-intervention group. These changes were restricted to the androgenreceptor pathway in the pea-intervention group, whereas the broccoli-intervention group also exhibited changes in several other pathways associated with prostate carcinogenesis, including the insulin-signaling, transforming growth factor $\beta$ and epidermal growth factor receptor pathways, in addition to changes in messenger RNA processing.

After further analyses, the authors suggest that isothiocyanates might affect these pathways via interaction with extracellular signaling peptides, rather than via intracellular activity. The effect of consumption of cruciferous vegetables on clinical end points, however, remains to be determined.

Original article Traka M et al. (2008) Broccoli consumption interacts with GSTM1 to perturb oncogenic signalling pathways in the prostate. PLoS ONE 3: e2568

\section{Efficacy of androgen signaling blockade in castration-resistant prostate cancer}

Evidence indicates that nongonadal androgen synthesis might persist and drive prostate cancer progression in men treated by castration. Attard et al. conducted a phase I trial to investigate the efficacy and safety of abiraterone acetate-which blocks cytochromeP17-mediated androgen biosynthesis-in the treatment of castration-resistant prostate cancer.

This open-label study enrolled 21 chemotherapy-naive men with hormone-refractory prostate cancer who had undergone castration. Patients were assigned to receive $250 \mathrm{mg}, 500 \mathrm{mg}, 750 \mathrm{mg}, 1,000 \mathrm{mg}$ or $2,000 \mathrm{mg}$ abiraterone acetate daily.

Circulating testosterone and dehydroepiandrosterone fell to undetectable levels in all patients within 8 days and 28 days of study initiation, respectively, whereas over 142 days, levels of adrenocorticotropic hormone increased by up to six times. In 14 (66\%) patients, $a \geq 30 \%$ decline in PSA levels was reported for least the first 3 months of follow-up; in other studies such a decline in PSA has been associated with a decreased risk of death from prostate cancer. Of the 11 patients who had pain that required analgesics at baseline, 8 had symptom improvement that permitted dose reduction or cessation. Most adverse events recorded were attributable to excess levels of mineralocorticoids, secondary to blockade of androgen synthesis.

The authors suggest that blockade of androgen signaling with abiraterone acetate could be a 